\title{
A handheld device for potential point-of-care screening of cancer $\dagger+$
}

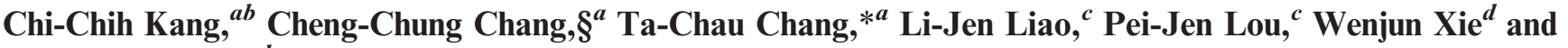 Edward S. Yeung ${ }^{d}$}

Received 5th December 2006, Accepted 29th May 2007

First published as an Advance Article on the web 8th June 2007

DOI: $10.1039 / \mathrm{b} 617733 f$

A simple handheld device based on the fluorescence analysis of 3,6-bis(1-methyl-4vinylpyridinium)carbazole diiodide (BMVC) stained cells was established for routine screening and potentially for early detection of cancer cells at extremely low cost. Flow cytometry assay further supported the utility of this simple device, where a preliminary study of tissue biopsy showed highly encouraging results.

\section{Introduction}

Cancer remains the leading cause of death worldwide: each year about 7 million people die from cancer, which is almost $12.5 \%$ of the total deaths in the world. A large amount of effort is therefore put into cancer treatment and diagnosis. Since early diagnosis plays a key factor in improving the survival of cancer, the development of a sensitive method for early detection is a major direction for cancer diagnosis. On the other hand, a point-of-care device would be very useful to health centers in the developing world. A simple, rapid, and non-invasive method at low cost for regular screening of cancer cells is another need for cancer diagnosis. Such a device may be particularly useful for tracing cancer recurrence. So, is it possible to develop a handheld device to bring hospital protocols to the home for the routine screening of cancer?

Recently, a fluorescence probe of 3,6-bis(1-methyl-4-vinylpyridinium)carbazole diiodide (BMVC) was synthesized for recognizing and stabilizing the G-quadruplex structure of the human telomeric sequence $\mathrm{d}\left(\mathrm{T}_{2} \mathrm{AG}_{3}\right)_{4} \cdot{ }^{1-4}$ In addition, bright fluorescence spots from BMVC could be observed in the nuclei of most H1299, CL1-0, HeLa, and Ca9-22 cancer cells, but were hardly found in the nuclei of MRC-5, IMR90, Detroit551 and NIH3T3 normal cells. ${ }^{5}$ This implied that the distinct fluorescence properties of BMVC can be used to distinguish cancer cells from normal cells. The present work aims at introducing a simple and rapid method for the routine screening of cancer cells at very low cost. In order to test the feasibility of

${ }^{a}$ Institute of Atomic and Molecular Sciences, Academia Sinica, P.O. Box 23-166, Taipei, Taiwan 106, Republic of China. E-mail: tcchang@po.iams.sinica.edu.tw

${ }^{b}$ Taiwan International Graduate Program and Department of Chemistry, National Tsing-Hua University and Academia Sinica, Taiwan, Republic of China

cDepartment of Otolaryngology, National Taiwan University Hospital and National Taiwan University College of Medicine, Taipei, Taiwan, Republic of China

${ }^{d}$ Ames Laboratory-USDOE and Department of Chemistry, Iowa State University, Ames, Iowa 50011, USA

$\uparrow$ Electronic supplementary information (ESI) available: video clips of fluorescence images for stained normal and cancer cells flowing through a PTFE tube. See DOI: 10.1039/b617733f

\$ The HTML version of this article has been enhanced with colour images.

$\S$ Present address: Department of Chemistry, National Chung-Hsin University, Taiwan. this simple device, a pilot ex vivo study was designed to identify malignant tumor cells from surgically resected tumors.

\section{Experimental}

\subsection{A handheld device for screening of cancer cells}

A handheld device includes a light emitting diode (LED) and a $470 \mathrm{~nm}$ band pass filter used for exciting the fluorescence probes and a $520 \mathrm{~nm}$ long pass filter used for eliminating background scattering. The fluorescence emitted from a sample could be visualized by the eye without using a detector. However, a detector such as a digital camera is recommended to record the images for further data analysis.

\subsection{Materials}

The synthesis of BMVC from 3,6-dibromo-9H-carbazole through the Heck reaction and methylation could be found elsewhere. $^{1,2}$ An MRC-5 human lung fibroblast, a H1299 human lung cancer and an NIH3T3 mouse embryonic fibroblast were purchased from ATCC (American Type Culture Collection) and grown under their complete growth mediums. A CL1-0 human lung cancer was kindly provided by Professor C. T. Chen at National Taiwan University.

\subsection{In vitro study}

Cells were grown in T25 flasks and harvested by trypsin EDTA. The harvested CL1-0 human lung cancer and NIH3T3 mouse fibroblast were separately stained by $1.5 \mu \mathrm{M}$ BMVC and $0.02 \mu \mathrm{M}$ acridine orange in the growth medium for $5 \mathrm{~min}$. The samples were centrifuged at $2500 \mathrm{rpm}$ for $5 \mathrm{~min}$ to remove the medium and then resuspended in phosphate buffered saline (PBS). A ca. $2 \mu \mathrm{L}$ cell suspension on a cover glass was examined using our simple device. The cell pellets, resuspended in a $500 \mu \mathrm{L}$ PBS with a cell density of $c a .2 \times 10^{5}$ cells $\mathrm{mL}^{-1}$, were further studied using flow cytometry. In addition, a 1/16" OD PTFE tube (purchased from VICI Valco Instruments Co. Inc.) was used for the flow experiments.

\subsection{A pilot ex vivo study}

A small piece of tumor was cut and preserved in Dulbecco's Modified Eagle's Medium (DMEM) immediately after 
resection. Then a cold knife was used to crumble the tissue. Cells in the culture medium were centrifuged at $1000 \mathrm{rpm}$ for $5 \mathrm{~min}$, and then resuspended with fresh culture medium. Equivalent volumes of cells were dropped onto three different slides and stained by trypan blue (TB), acridine orange (AO), and BMVC, respectively. Observation for $\mathrm{TB}$ under a light microscope was applied to confirm the cell viability. Using our simple device, AO staining was used to estimate the total cell numbers under screening and BMVC staining was assessed for screening of the cancer cells. This study was approved by the Institutional Review Board at the National Taiwan University Hospital (No. 9561709041) and informed consent was obtained from all participants.

\section{Results and discussion}

\subsection{A simple and rapid method for screening cancer cells}

Many people dream of a handheld device for instant diagnosis of cancer or other diseases. ${ }^{6}$ To miniaturize the diagnostic device, we have to figure out what is necessary and what can be scaled down. We have demonstrated a cell-based microarray to measure the detection limit of cancer cells upon incubation with BMVC. ${ }^{7}$ To excite the fluorescence probe, a light source is required. We found that a light emitting diode (LED) is sufficient for our purpose. It is much cheaper and smaller than a laser. The next question is to optimize the signal/noise ratio. In order to eliminate background scattering, a $470 \mathrm{~nm}$ band pass filter, characterized by the absorption of the fluorescence probe, is introduced in front of the sample and a $520 \mathrm{~nm}$ long pass filter, characterized by the emission of the fluorescence probe, is used. Fig. 1(a) shows this simple device. The most prominent feature of this handheld device is its compactness and can be carried by the user for performing a test even at home. In addition, this device does not involve any sophisticated equipment and can therefore easily be constructed in-house.

In order to show our results and for further data analysis, a digital camera is applied to record the fluorescence images. Fig. 1(b-d) shows three sets of fluorescence images of CL1-0 cancer cells, NIH3T3 normal cells, and their mixture with a $1: 10 \mathrm{C} / \mathrm{N}$ cell ratio stained by $0.02 \mu \mathrm{M}$ AO (upper) and $1.5 \mu \mathrm{M}$ BMVC (lower), respectively. The non-fixed cells placed on a glass slide were illuminated by an LED and the fluorescence images were collected by a conventional digital camera. The images showed a brighter fluorescence of BMVC in the cancer cells than in the normal cells. The brighter fluorescence intensity could be used as the criterion for cancer detection. However, the very small fraction of normal cells with emitting BMVC fluorescence could make such a diagnosis of cancer ambiguous.

To improve the reliability of this method, another criterion for cancer diagnosis was introduced by comparing the ratio of the number of the cells with BMVC fluorescence to the total number of the cells under examination. Considering the rapid staining of AO to both cancer cells and normal cells, ${ }^{7,8}$ we then used AO to stain the cells for estimating the total number of cells. Fig. 1(b) shows that there are $c a$. 150 cells stained by AO and $c a$. 22 cells stained by BMVC in the CL1-0 cells. On the other hand, there are 4 cells with weak fluorescence stained by (a)

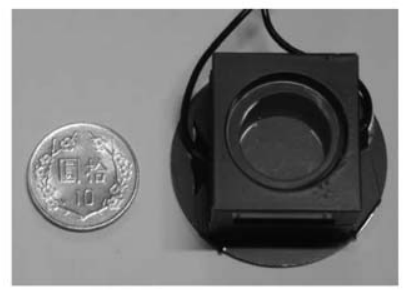

(b)

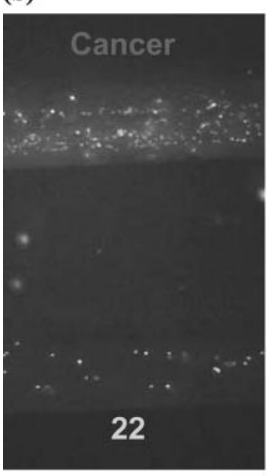

(c)

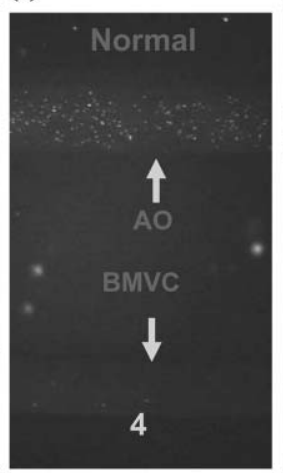

(d)

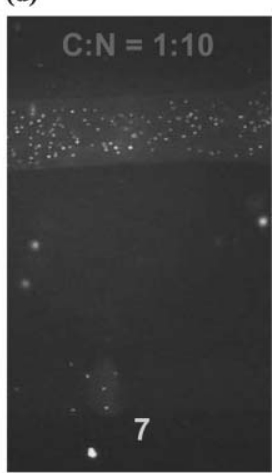

(e)

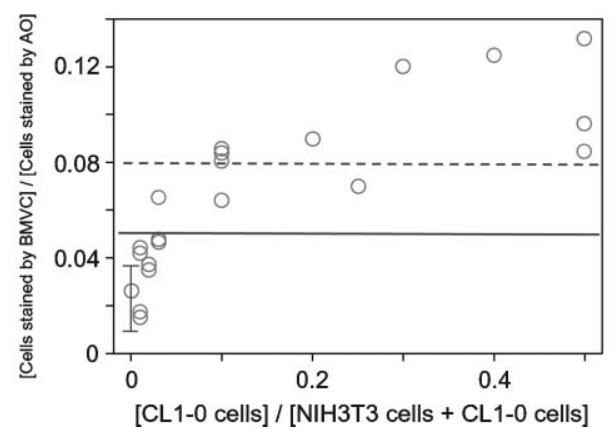

Fig. 1 (a) Handheld device for detecting cancer cells - a coin is used to illustrate the compactness of this device. (b) Fluorescence images of CL1-0 cancer cells, (c) NIH3T3 normal cells, and (d) their $1: 10$ mixture stained by $0.02 \mu \mathrm{M}$ AO (upper) and $1.5 \mu \mathrm{M}$ BMVC (lower) for $5 \mathrm{~min}$. These pictures were collected by a color camera under LED illumination. (e) Statistical histogram of the cell ratio based on BMVC/ AO fluorescence of the CL1-0 cells to the sum of NIH3T3 and CL1-0 cells. The solid line represents a baseline for follow-up examination and the dashed line is the recommended threshold for follow-up examination.

BMVC in the NIH3T3 normal cells, as shown in Fig. 1(c). In the mixture of NIH3T3 normal cells and CL1-0 cancer cells with cell ratio of $10: 1$, respectively, Fig. 1(d) shows 4 cells with relatively bright fluorescence and 3 cells with weak fluorescence. Note that the experimental conditions were optimized by varying the BMVC concentration and its incubation time to detect sufficient BMVC fluorescence from the CL1-0 cells with minimal BMVC fluorescence from the NIH3T3 cells. Under $1.5 \mu \mathrm{M}$ BMVC with a 5 min incubation time, only about $15 \%$ of the CL1-0 cells showed BMVC fluorescence. A statistical histogram of the cell ratio based on BMVC/AO fluorescence of the CL1-0 cells to the sum of NIH3T3 and CL1-0 cells is shown in Fig. 1(e). Each data point represents an average of 5-10 slides measured from a single sample and more than 10 sample sets for the pure NIH3T3 were measured. Although the precise cutoff for accurate 
detection of cancer cells has not been determined in this case, we suggest that a cell ratio stained by BMVC/AO larger than 0.05 [the solid line in Fig. 1(e)] represents a baseline for followup examination, and a ratio larger than 0.08 (dashed line) definitely requires more detailed examination by other methods. Note that the background is defined as the ratio of normal cells stained by BMVC to normal cells stained by AO. Here we found that the mean background is around 0.025 and the standard deviation is $c a$. 0.01 from measurements repeated at least ten times. We further took the 0.01 ratio of cancer cells to normal cells as a critical level for the possible false detection. Accordingly, our simple device could detect the ratio of CL1-0 cancer cells to the NIH3T3 fibroblast at 0.1 for a cell ratio stained by BMVC/AO at 0.08 , and possibly 0.05 for a cell ratio stained by BMVC/AO at 0.05 . Nevertheless, our results suggest that this simple device based on the distinct cell-based $\mathrm{BMVC} / \mathrm{AO}$ fluorescence is useful for screening cancer cells.

\subsection{Evaluation of the simple method by flow cytometry}

To further evaluate the feasibility of our simple device for selfscreening of cancer cells, we must compare the results obtained from our simple device with an established method. Flow cytometry was originally developed for measuring the optical or fluorescence characteristics of cells or particles in suspension. ${ }^{9}$ Recently, it has become an important method in cancer research for monitoring tumor progression, ${ }^{10}$ the screening of anti-cancer drugs, ${ }^{11}$ and clinical diagnosis. ${ }^{12}$ Schmidt et al. ${ }^{10}$ showed that flow cytometry can be used for the accurate detection of as few as one fluorescent tumor cell per 200000 host cells in dissociated tissues by using a GFP-transfected breast carcinoma cell line. In our work, we simply apply flow cytometry to validate our simple device for the discrimination of cancer cells from normal cells in a mixture.

Here, the H1299 human lung cancer and MRC-5 human lung fibroblast were separately incubated with $2 \mu \mathrm{M}$ BMVC and $2 \mathrm{nM} \mathrm{AO}$ for $5 \mathrm{~min}$. The cell pellets obtained from centrifugation were resuspended in $500 \mu \mathrm{L}$ PBS. A minimum of $1 \times 10^{5}$ cells per sample were first analyzed by using a flow cytometer (Becton Dickinson FACS Canto) excited at $488 \mathrm{~nm}$, and emission was collected through band pass filters centered at 530/30 (FITC channel) for AO and 575/26 nm (PE channel) for BMVC. Following the gating region on the forward scatter characteristic versus side scatter characteristic set, we first excluded cell aggregates and any debris. Fig. 2(a) and 2(b) show the dot plots of the pure MRC-5 cells and the mixture of H1299 and MRC-5 cells at a ratio of $1: 100$ stained by both $\mathrm{AO}$ and BMVC, respectively. Here the dot plots are separated to four quadrants, where Q3 represents cells without staining, in Q4 are cells with only AO positive staining, and in Q2 are cells with both $\mathrm{AO}$ and BMVC positive staining. We simply put our attention on the ratio of Q2 to the total number cells for each sample. Flow cytometry analysis showed that there is $1.4 \%$ BMVC positive for pure MRC-5 cells and 3.7\% for the mixture at $1: 100$. In addition, we found that the average BMVC positive brightness in cancer cells was about twice that of normal cells. Fig. 2(c) shows the plots of the BMVC positive signal versus the ratio of the cancer cells to the total sum of cancer and normal cells. Again, the background is defined as the BMVC positive signal for pure normal cells. Here we took the BMVC positive signal at $2 \%$ as a threshold for the possible false detection. Thus, our data suggest that we could detect at least the cell ratio of cancer cells to normal cells at $1: 100$, and possibly down to $1: 1000$. It should be noted that AO is simply used to stain both normal and cancer cells as a reference for BMVC in our simple device - it is not necessary in flow cytometry measurements. Nevertheless, the flow cytometry results confirmed the feasibility of BMVC in our simple device for cancer diagnosis.

\subsection{A preliminary study of tissue biopsy}

In order to test the feasibility of this handheld device in clinical application, a pilot ex vivo study was designed to identify malignant tumor cells from surgically resected tumors. A total of 14 tumor samples obtained from the head and neck regions was examined. One sample was regarded as inadequate due to insufficient viable cells for evaluation. There were 11 malignant tumors in the remaining 13 samples. All of these 11 samples showed positive BMVC staining, while the other two benign tumor cells were negative for BMVC staining.

Fig. 3(a) and 3(b) show fluorescence images of $\mathrm{AO}$ and BMVC stains of a sample collected from a 63-year-old man with a $1.5 \times 2 \mathrm{~cm}$ tumor over the left tongue border. Our data (a)

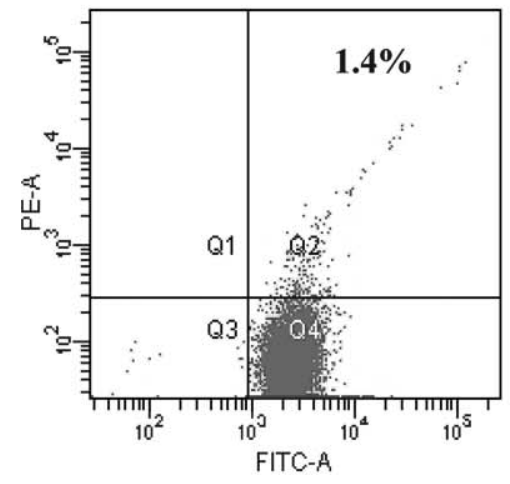

(b)

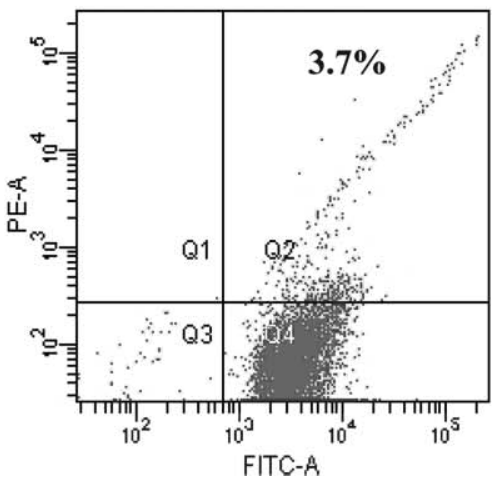

(c)

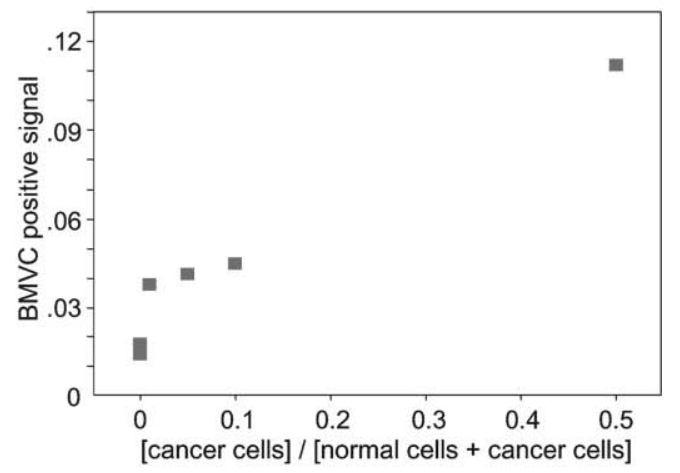

Fig. 2 Detection of cancer cells by flow cytometry. Analysis of the dot plots of (a) MRC-5 only, and (b) the mixture of MRC-5 and H1299 cells at a ratio of 100 to 1 , stained by $2 \mathrm{nM}$ AO and $2 \mu \mathrm{M}$ BMVC for $5 \mathrm{~min}$. The percentage in each dot plot shows the percentage of BMVC positive signal (Q2 region) to total cells. (c) Statistical histogram of BMVC positive signal versus the ratio of the cancer cells to the total number of cells. 
(a)

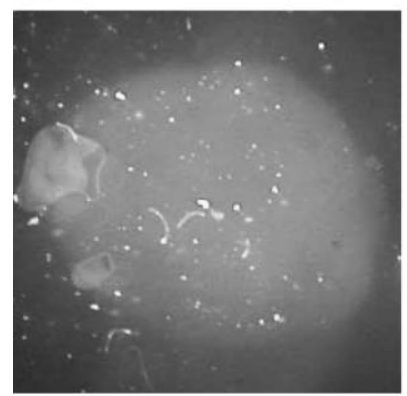

(c)

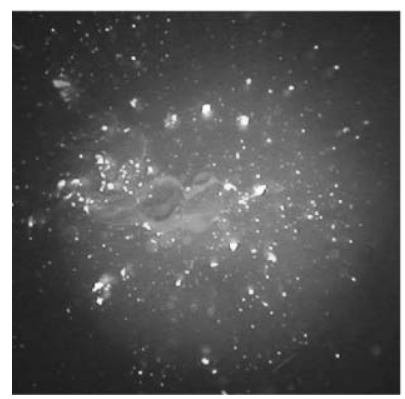

(b)

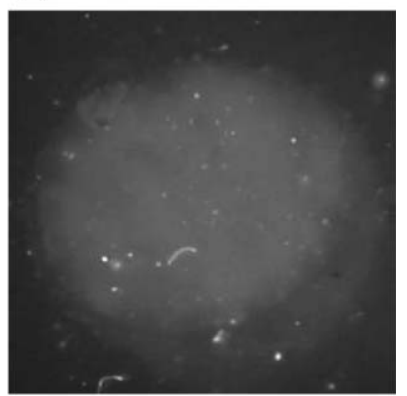

(d)

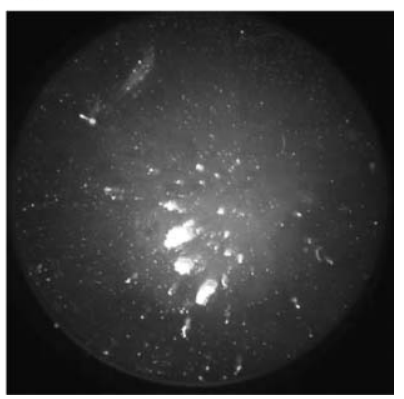

Fig. 3 Fluorescence images of (a) AO and (b) BMVC stains revealed sufficient cells with negative BMVC stain, for proposal as a benign tumor of the tongue by using our handheld device. Fluorescence images of (c) AO and (d) BMVC stains revealed sufficient cells with positive BMVC stain, for proposal as a squamous carcinoma of the tongue.

revealed sufficient cells with negative BMVC stain. The pathology showed a squamous papilloma, which is a benign tumor of the tongue. On the other hand, a typical positive example of the AO and BMVC stains is shown in Fig. 3(c) and 3(d), respectively. The pathology showed a squamous cell carcinoma for this case. The high correlation of our analysis with the standard pathological method illustrated that this simple method is feasible for cancer diagnosis. Although the preliminary test of 14 cases is not enough to draw a solid conclusion, it is sufficient to indicate a promising approach for clinical applications. In addition, the test result is immediately available to the user right after the test has been conducted.

A test for cancer must be exquisitely accurate; otherwise, many patients without cancer may test positive. The detection of a few cells with bright BMVC fluorescence may serve to identify patients at risk of developing cancer and therefore deserves further diagnosis by a physician. In addition, the response of cancer cells and normal cells to the probe molecule could vary considerably from one organ system to another. To explore this simple approach, we plan to examine certain cancer cells that can be easily obtained, such as from skin, oral, bodily fluids or even cervix, and fine needle aspiration, etc. Considering the extremely low cost for each test, this simple and rapid method is available and affordable for the routine pre-screening of cancer.

\subsection{Detection limit}

Early diagnosis is the key for better treatment of cancer and therefore improves the survival of the patient. In addition, (a)

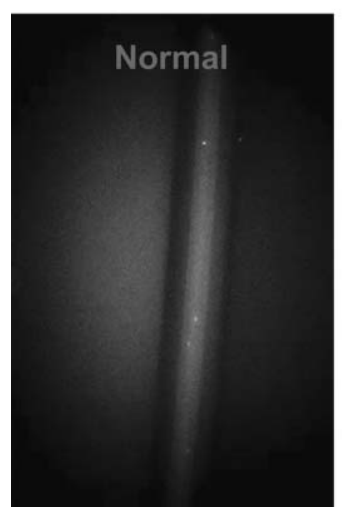

(b)

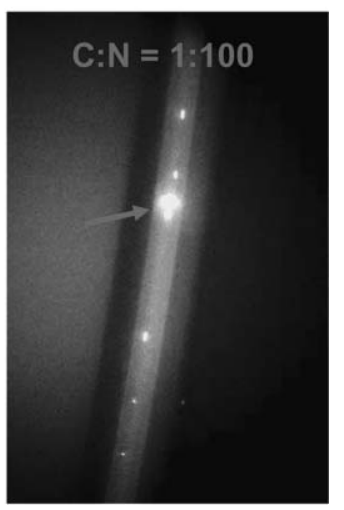

Fig. 4 Fluorescence image of (a) NIH3T3 normal cells stained by BMVC flow through a tube, and (b) a typical bright fluorescence aggregate in the mixture of NIH3T3 normal cells and CL1-0 cancer cells with the cell ratio $100: 1$ stained by BMVC. These data were obtained using our handheld device.

recurrent tumors are the major killer of cancer patients. A simple and low cost method is invaluable for routine testing to follow up on the recurrence of cancer. Could we use this simple device for the detection of a limited number of cancer cells? Here we take the cell aggregation as an advantage and propose a simple method for possible detection of cancer cells at an early stage by the bright BMVC fluorescence from clusters. Using our simple device, Fig. 4(a) and 4(b) show the images of pure NIH3T3 cells and the mixture of CL1-0 and NIH3T3 at a cell ratio of $1: 100$, respectively, stained by BMVC in a PTFE tube. We estimated the total number of cells that flowed through the tube to be $c a$. 5000. A typical video of the cells flowing in the tube can be found in the ESI. $\dagger$ The striking feature was that several clusters with very bright fluorescence were found in the mixture of NIH3T3 and CL1-0 cells. The key finding is that up to now such bright BMVC fluorescence was not observed in the NIH3T3 cells. This cluster is due to aggregation of at least three cancer cells. Moreover, if the flow rate is $1 \mathrm{~cm} \mathrm{~s}^{-1}$, it takes less than $20 \mathrm{~min}$ to run $1 \mathrm{~mL}$ of cells. The analysis time can be shortened when several tubes are running simultaneously. Although this approach depends upon the possibility of cell aggregation, we consider that the simple approach by monitoring such bright BMVC fluorescence in the $100: 1$ cell mixture illustrates the feasibility for an early detection of cancer cells. It is suggested that the detection of such bright fluorescence from cells that flow through a tube deserves further diagnosis by a physician. This simple approach is particularly useful for fine needle aspiration for the test of outpatients.

\section{Conclusions}

We have described a very simple, rapid and non-invasive method for the screening of cancer cells. This method could substantially accelerate the clinical analysis after the samples are obtained from tissue biopsy, needle biopsy, or bodily fluids, etc. In addition, the cost of the device including a conventional camera and a small centrifuge would be several hundred US dollars, but the cost of each run is less than US 
$\$ 10$, making this device cheap enough for routine cancer screening. Moreover, this method forms a new and important basis for the possible development of novel assays to bring the hospital device to the home for routine pre-screening of cancer cells, especially for the follow-up of cancer patients. In addition, a number of potential refinements can be incorporated into future studies, such as coupling to a capillary tube for single cancer cell detection, ${ }^{13}$ to a fiber-optic detector for spectroscopic identification, ${ }^{14}$ and as a microarray-based technique for the high throughput screening. ${ }^{15}$

\section{Acknowledgements}

T.-C. C. thanks the support from Academia Sinica (AS-95-TPAB2) and the National Science Council of the Republic of China (Grant NSC-94-2113-M001-047).

\section{References}

1 C. C. Chang, J. Y. Wu and T.-C. Chang, J. Chin. Chem. Soc., 2003, 50, $185-188$.

2 C.-C. Chang, J.-Y. Wu, C.-W. Chien, W.-S. Wu, H. Liu, C.-C. Kang, L.-J. Yu and T.-C. Chang, Anal. Chem., 2003, 75, 6177-6183.
3 C.-C. Chang, I.-C. Kuo, I.-F. Ling, C.-T. Chen, H.-C. Chen, P.-J. Lou, J.-J. Lin and T.-C. Chang, Anal. Chem., 2004, 76, 4490-4494.

4 C.-C. Chang, J.-F. Chu, F.-J. Kao, Y.-C. Chiu, P.-J. Lou, H.-C. Chen and T.-C. Chang, Anal. Chem., 2006, 78, 2810-2815.

5 C.-C. Chang, I.-C. Kuo, J.-J. Lin, Y.-C. Lu, C.-T. Chen, H.-T. Back, P.-J. Lou and T.-C. Chang, Chem. Biodiv., 2004, 1, 1377-1384

6 J. Kling, Nat. Biotechnol., 2006, 24, 891-893.

7 C. C. Kang, C. C. Chang, J. Y. Cheng and T.-C. Chang, J. Chin. Chem. Soc., 2005, 52, 1069-1072.

8 D. M. Neville and D. F. Bradley, Biochim. Biophys. Acta, 1961, 50, 397.

9 M. Brown and C. Wittwer, Clin. Chem., 2000, 46, 1221-1229.

10 C. M. Schmidt, S. L. Settle, J. L. Keene, W. F. Westlin, G. A. Nickols and D. W. Griggs, Clin. Exp. Metastasis, 1999, $17,537-544$

11 M. R. Clutter, P. O. Krutzik and G. P. Nolan, Drug Discovery Today, 2005, 2, 295-302.

12 N. Wedemeyer and W. N. Pötter, Clin. Genet., 2001, 60, 1-8.

13 D. Isailovic, H. W. Li, G. J. Phillips and E. S. Yeung, Appl. Spectrosc., 2005, 59, 221-226.

14 U. Utzinger and R. R. Richards-Kortum, J. Biomed. Opt., 2003, 8, 121-147.

15 M. H. Yen, J. Y. Cheng, C. W. Wei, Y. C. Chuang and T. H. Young, J. Micromech. Microeng., 2006, 16, 1143-1153. 